\title{
Nouvelle méthode continue pour la mesure objective de la rigidité des gels lactés
}

\author{
par \\ J. P. RAMET*, E. EL MAYDA*, F. WEBER*
}

\section{Ré s u m é}

L'évolution de la rigidité de différents types de coagula lactés en cours de formation a été suivie en mesurant la force exercée au niveau d'une petite sphère métallique $(\varnothing 15 \mathrm{~mm})$ immergée dans le lait et soumise à un mouvement vertical oscillant de faible amplitude $(5 \mathrm{~mm})$ et de basse fréquence $\left(8,3 \times 10^{-3} \mathrm{~Hz}\right)$. Ce mobile a été adapté à une machine universelle d'essai Instron, équipée d'une cellule de mesure, de capacité $0-5 \mathrm{~N}$ et d'un enregistreur graphique permettant de suivre la courbe force/temps.

Pour différents laits traités, soit par voie enzymatique à l'aide de présure de veau (P) ou de la protéase neutre de Bacillus subtilis (BS), soit par voie acide à l'aide de bactéries lactiques thermophiles (BL), soit par addition de carraghénates (C), une déviation sinusoïdale faible et régulière de l'enregistrement a été observée lorsque le lait est à l'état liquide. Au contraire, lorsque le processus de gélification commence, la déviation s'accroît progressivement. Les points correspondant à l'amplitude maxima atteinte à chaque demi-période se situent sur deux courbes, dont l'allure sigmoïde est caractéristique du comportement rhéologique propre à chacun des gels lactés.

En raison de sa grande sensibilité $\left(2.10^{-4} \mathrm{~N}\right)$, de sa bonne répétabilité $(\mathrm{V}=1,88 \%)$ et de son caractère non destructif, la méthode proposée apparaît bien adaptée à la mesure fine des propriétés rhéologiques des gels fragiles de biopolymères. Des développements sont poursuivis au laboratoire pour l'étude des facteurs influençant les processus de gélification et pour la mise au point d'un capteur spécifique destiné à l'industrie.

* Institut National Polytechnique de Lorraine, Laboratoire de Technologie Laitière, 32, rue Sainte-Catherine, 54000 Nancy. 


\section{Su m mary}

\section{A NEW CONTINUOUS METHOD FOR OBJECTIVE MEASUREMENT OF RIGIDITY OF MILK GELS}

A Instron Universal Testing Machine has been adapted for curd tension studies of milk gels. The rigidity of curd was measured by means of a metallic sphere $(\varnothing 5 \mathrm{~mm}$ ) which oscillated vertically and slowly through a small amplitude $(5 \mathrm{~mm})$ at $4 \mathrm{~cm}$ below the surface of milk. The strength was quantified in standart units $(N)$ by a load cell connected on sensing unit. Typical sigmoïd curves of the evolution of gels strength versus time were obtained with four types of milk curds settled either from clotting enzymes (rennet and bacterial protease from Bacillus subtilis), either from lactic starters, either from carrageenan. Precision of the new method $\left(2.10^{-4} \mathrm{~N}\right)$ and its reliability (variation coefficient : 1,88\%) were very satisfactory. Practical applications on laboratory and industrial fields are further investigated.

\section{INTRODUCTION}

La mesure de la rigidité des gels lactés possède en Technologie Laitière un triple intérêt. Elle permet tout d'abord de déterminer l'aptitude coagulante des laits et celle de certains auxiliaires de fabrications (enzymes coagulantes, gélifiants) ; elle permet également de définir les caractéristiques rhéologiques et texturales des produits finis ou en cours d'élaboration qui sont une composante essentielle de leur qualité. Enfin, elle constitue un outil de recherche fondamentale privilégié pour étudier les mécanismes de la coagulation. De multiples méthodes instrumentales ont été développées dans ce but au cours des vingt dernières années; leurs principes, leurs applications ont fait l'objet de nombreuses publications dont la synthèse a été faite par Thomasow et Voss, 1977.

Il ressort de l'ensemble de ces travaux qu'une difficulté majeure dans l'appréciation objective de la tension des gels résulte de leur grande fragilité et de leur propension à la synérèse spontanée. De ce fait, les méthodes non destructives apparaissent le mieux adaptées et ont été les plus développées à partir de principes pourtant très différents: viscosimètres de torsion (Burnett et Scott Blair, 1963; Frentz, 1965 ; Gervais et Cerf, 1982), pendules oscillants (Zannoni et Annibaldi, 1981), transmetteurs de pression (Vanderheiden, 1976; Rowalchyk et Olson, 1978).

Les études réalisées avec ces appareils au laboratoire et dans l'industrie sont intéressantes et ont permis notamment de mieux connaître l'importance respective des différents paramètres de coagulation (Tarodo de la Fuente et Frentz, 1966; Schalinatus et Behnke, 
1967 ; Ramet et Weber, 1980 ; Ramet et al., 1982), mais la comparaison des résultats obtenus à l'aide des différentes techniques est rarement possible car ceux-ci sont exprimés en unités arbitraires spécifiques à chaque instrument et difficilement convertibles en grandeurs physiques exactes.

Dans la présente communication, nous exposerons le principe d'une méthode de mesure originale ainsi que son application à l'aide d'une machine universelle d'essais Instron.

\section{MATERIELS ET METHODES}

\section{Coagulation du lait}

La rigidité de trois types de coagula lactés en cours de formation a été suivie en fonction du temps. Un substrat constitué de $300 \mathrm{~g}$ de lait écrémé préalablement reconstitué à partir d'une poudre "basse température " a été placé dans un réacteur en verre (hauteur : $16 \mathrm{~cm}$, diamètre : $8 \mathrm{~cm}$ ) et maintenu à température contrôlée par circulation d'eau dans la double paroi. La coagulation du lait a été réalisée soit par voie enzymatique à l'aide de présure de veau $(P)$ provenant des Laboratoires Carlin Marshall ou de la protéase neutre de Bacillus subtilis (BS), préparée par la Société Gist-Brocades, soit par voie acide à l'aide de bactéries lactiques (BL) thermophiles commercialisées par les Laboratoires Visby (mélange équicellulaire de Streptococcus thermophilus et de Lactobacillus bulgaricus), soit par addition de carraghénates (C) élaborés par la Société Satia. Les conditions particulières de coagulation sont données avec les résultats dans la légende des figures.

\section{Mesure de la rigidité du gel}

La rigidité des différents types de gels a été suivie au cours du temps en mesurant la force exercée au niveau d'une petite sphère $(\varnothing: 15 \mathrm{~mm})$ en acier chromé soumise à un mouvement vertical sinusoïdal de faible amplitude $(5 \mathrm{~mm})$ et de basse fréquence $\left(8,3 \cdot 10^{-3} \mathrm{~Hz}\right)$. Cette sphère a été adaptée par l'intermédiaire d'une tige rigide (L. : $200 \mathrm{~mm}-\varnothing: 2 \mathrm{~mm}$ ) à une machine universelle d'essai Instron 1122 équipée d'une cellule de mesure de force de capacité $0-5 \mathrm{~N}$ et connectée à un enregisteur graphique permettant de visualiser l'évolution de la force mesurée en fonction du temps. L'ensemble du dispositif de mesure est schématisé sur la figure 1.

Les caractéristiques géométriques du mobile et de la cinématique ont été choisies pour éviter toute turbulence du lait et tout cisaillement du gel; dans ces conditions, la cohésion et l'adhérence du coagulum sur le métal sont satisfaisantes. 
MACHINE UNIVERSELLE D’ESSAIS

INSTRON 1122

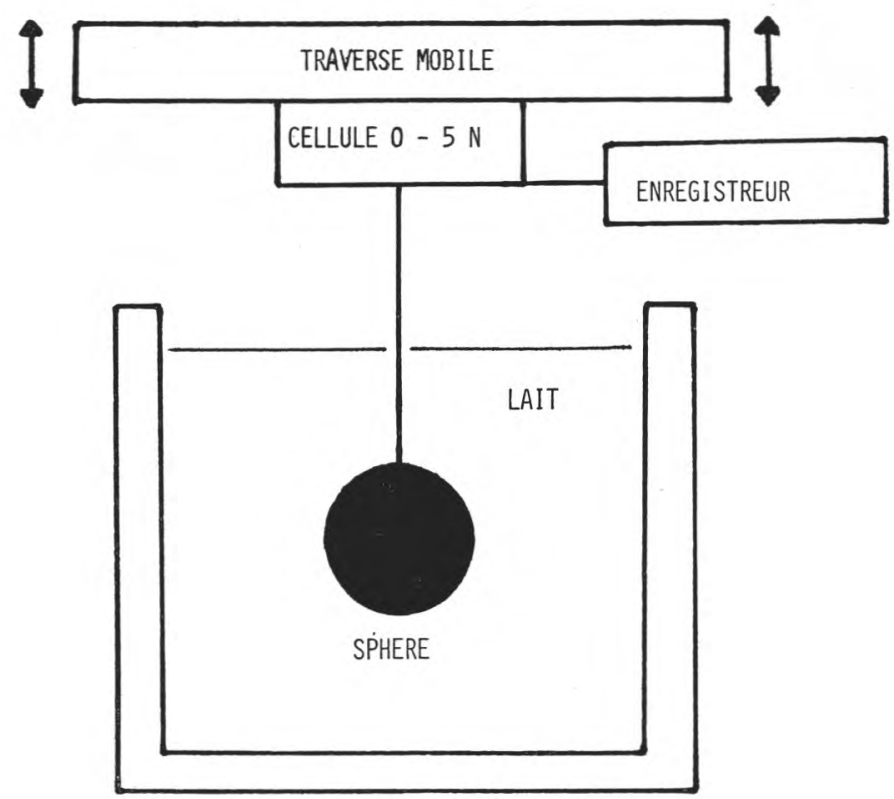

fig. 1

Schéma du dispositif de mesure utilisé.

Schematic drawing of the measuring system.

Par ailleurs, la masse $(21 \mathrm{~g})$ et la vitesse du mobile $(5 \mathrm{~mm} / \mathrm{mn})$ ont été définies pour interdire tout effet inertiel venant interférer avec la force propre exercée par le gel.

Après ajout de l'agent coagulant, le mobile a été immergé et le centre de la sphère placé très exactement à $4 \mathrm{~cm}$ sous la surface du lait; l'asservissement du mouvement oscillant a été ensuite ajusté à $\pm 2,5 \mathrm{~mm}$ de part et d'autre de ce point moyen.

\section{RESULTATS}

L'analyse des différentes courbes obtenues (fig. 2 à 5) révèle deux phases distinctes selon l'état physique du milieu. Initialement, lorsque le lait est liquide, le tracé est sinusoïdal, d'amplitude faible 


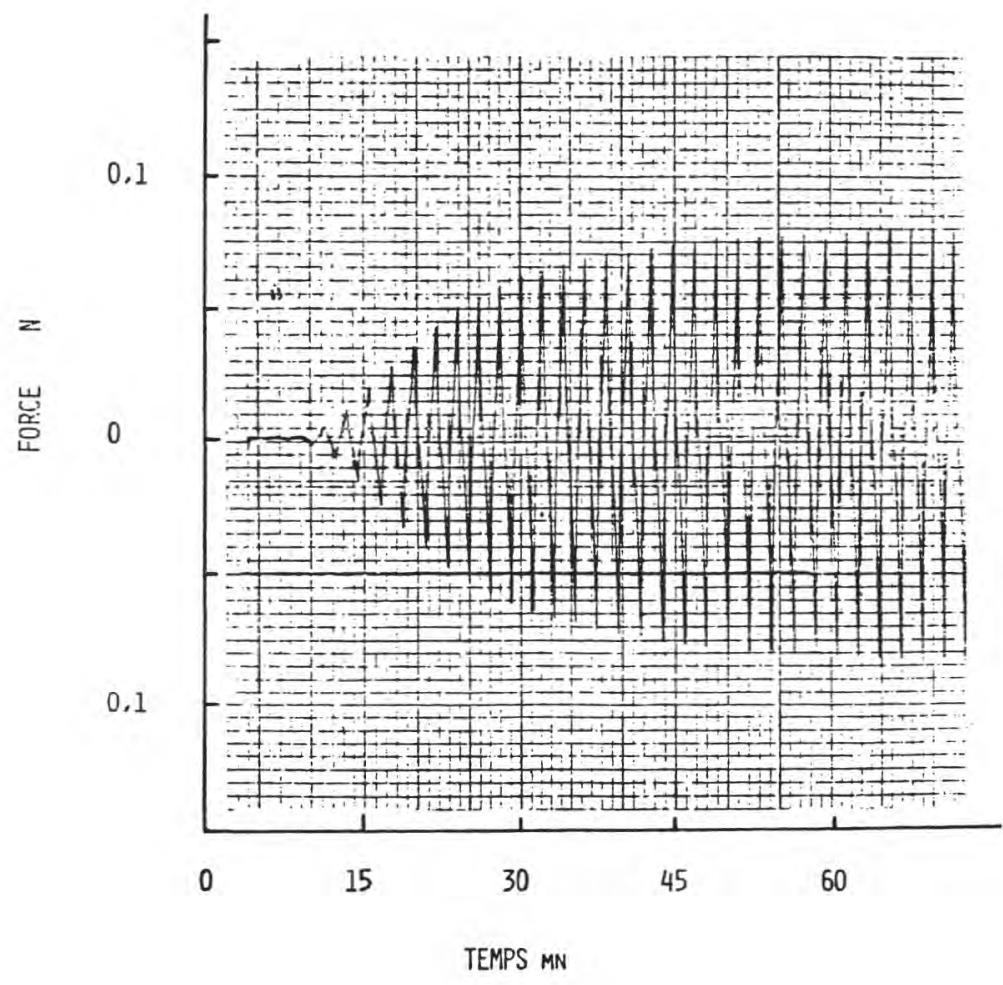

fig. 2

Evolution de la rigidité d'un gel obtenu à l'aide de présure Conditions de coagulation :

Substrat : lait écrémé reconstitué ; matière sèche : $10 \%$; $\mathrm{CaCl}_{2}, 2 \mathrm{H}_{2} \mathrm{O}$ : $10 \mathrm{~g} / 100 \mathrm{~kg}$ lait ; température : $35^{\circ} \mathrm{C}$; présure : $30 \mathrm{ml} / 100 \mathrm{~kg}$ lait.

Evolution of rigidity of rennet-induced skim-milk gel

Clotting conditions:

Substrate: reconstituted skim-milk; dried matter: 10\%; $\mathrm{CaCl}_{2}, 2 \mathrm{H}_{2} \mathrm{O}$ : $10 \mathrm{~g} / 100 \mathrm{~kg}$ of milk; temperature: $35^{\circ} \mathrm{C}$; rennet: $30 \mathrm{ml}$ $100 \mathrm{~kg}$ of milk.

et constante. Il apparaît que cette amplitude est fonction de la viscosité du milieu étudié, celle-ci étant en effet plus élevée pour un lait de $15 \%$ de matière sèche (fig. 4) que pour un lait à $10 \%$ d'extrait sec (fig. 3). Par ailleurs, lorsque le déplacement du mobile est réalisé dans l'air, la déviation observée est nulle et le tracé rigoureusement rectiligne; cette dernière observation confirme 


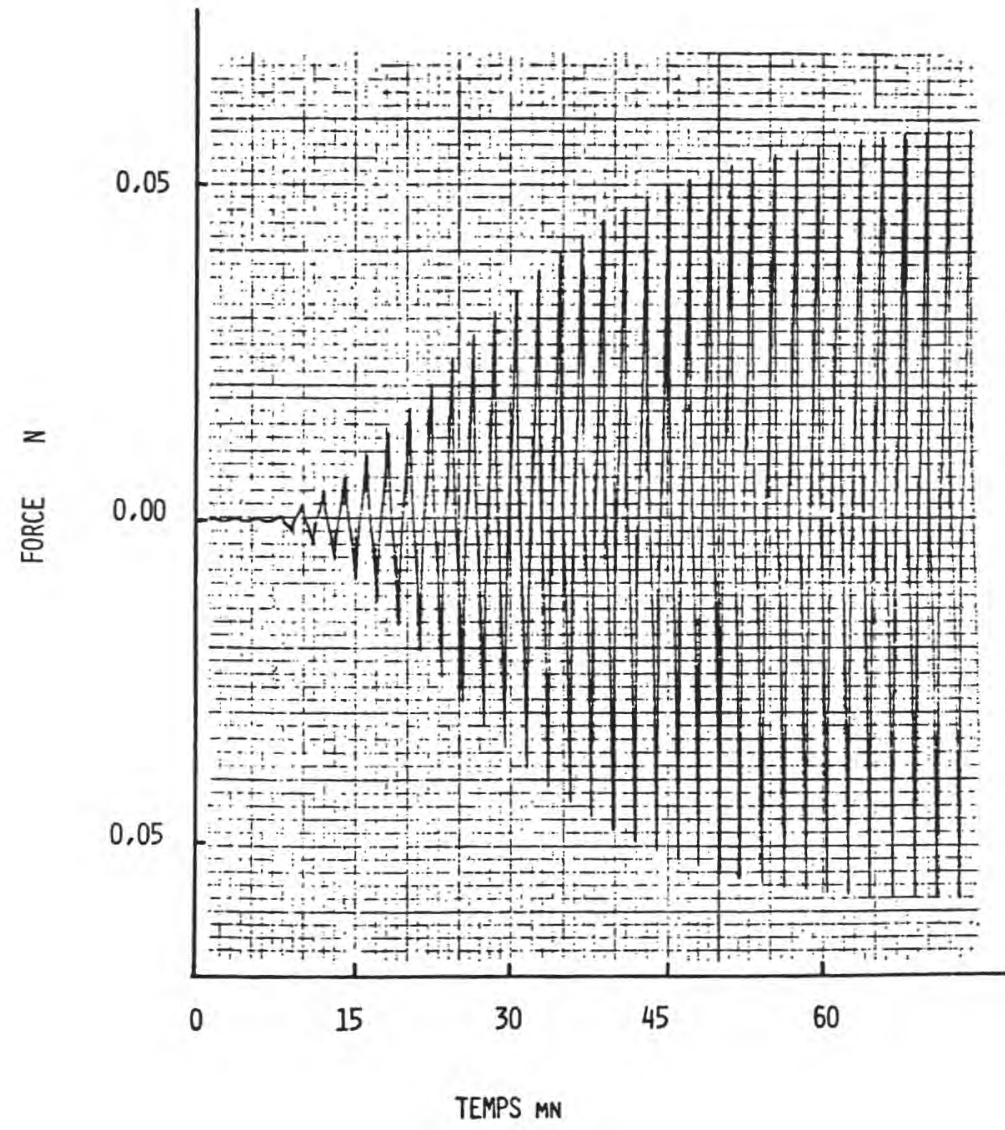

fig. 3

Evolution de la rigidité d'un gel obtenu à l'aide de la protéase neutre Bacillus subtilis

Conditions de coagulation :

Substrat : lait écrémé reconstitué ; matière sèche : $10 \% ; \mathrm{CaCl}_{2}, 2 \mathrm{H}_{2} \mathrm{O}$ : $10 \mathrm{~g} / 100 \mathrm{~kg}$ lait ; température : $35^{\circ} \mathrm{C}$; protéase : $6 \mathrm{~g} / 100 \mathrm{~kg}$ lait.

Evolution of rigidity of skim-milk gel induced by Bacillus subtilis neutral protease

Clotting conditions:

Substrate: reconstituted skim-milk; dried matter: $10 \%$; $\mathrm{CaCl}_{2}, 2 \mathrm{H}_{2} \mathrm{O}$ : $10 \mathrm{~g} / 100 \mathrm{~kg}$ of milk; temperature: $35^{\circ} \mathrm{C}$; protease: $6 \mathrm{~g} / 100 \mathrm{~kg}$ of milk. 


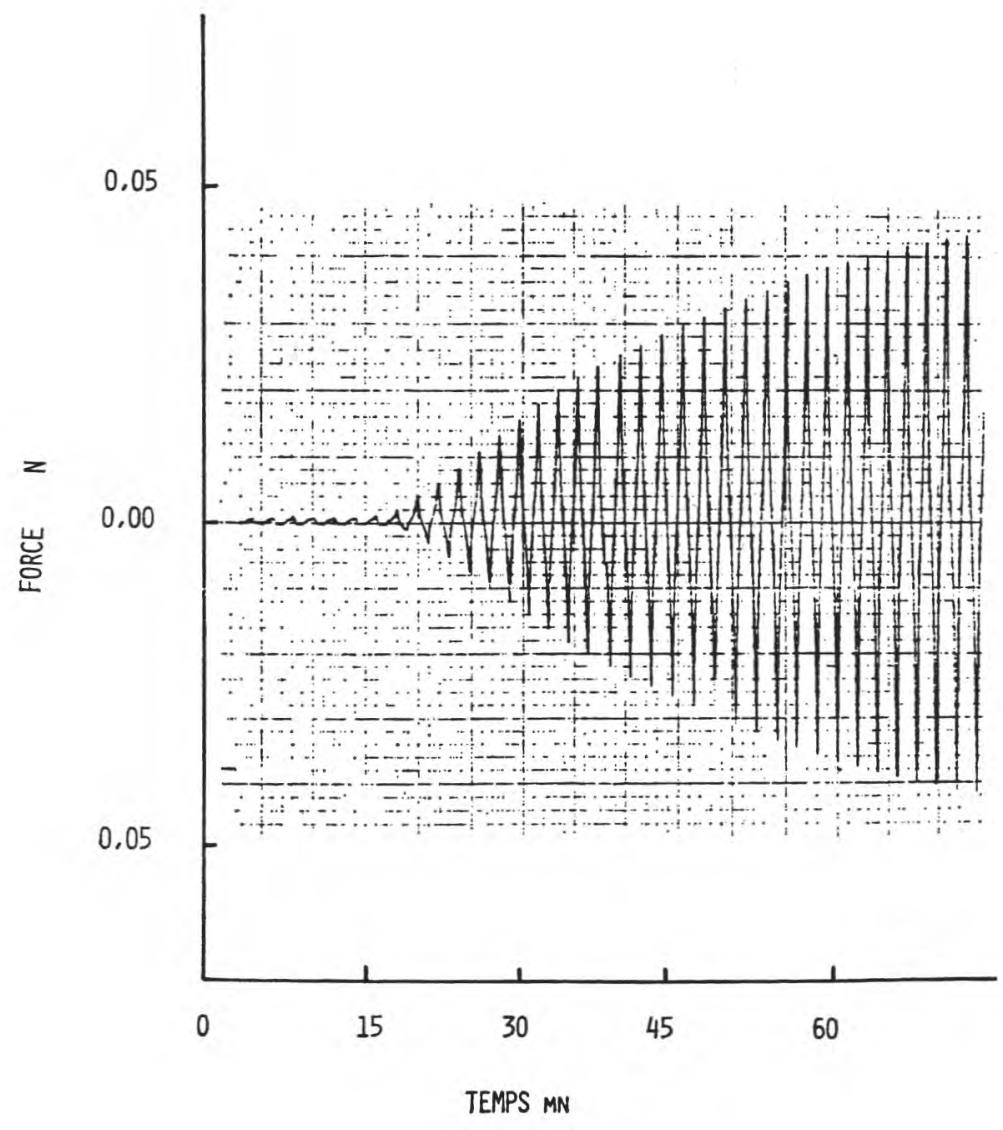

fig. 4

Evolution de la rigidité d'un gel obtenu par acidification lactique Conditions de coagulation :

Substrat : lait écrémé reconstitué ; matière sèche : $15 \% ; \mathrm{CaCl}_{2}, 2 \mathrm{H}_{2} \mathrm{O}$ : $10 \mathrm{~g} / 100 \mathrm{~kg}$ lait ; température : $35^{\circ} \mathrm{C}$; levains lactiques thermophiles : $10 \mathrm{~kg} / 100 \mathrm{~kg}$ lait.

Evolution of rigidity of skim-milk gel induced by acidification Clotting conditions:

Substrate: reconstituted skim-milk; dried matter: $10 \% ; \mathrm{CaCl}_{2}, 2 \mathrm{H}_{2} \mathrm{O}$ : $10 \mathrm{~g} / 100 \mathrm{~kg}$ of milk; temperature: $35^{\circ} \mathrm{C}$; lactic thermophilic bacteria: $10 \mathrm{~g} / 100 \mathrm{~kg}$ of milk. 


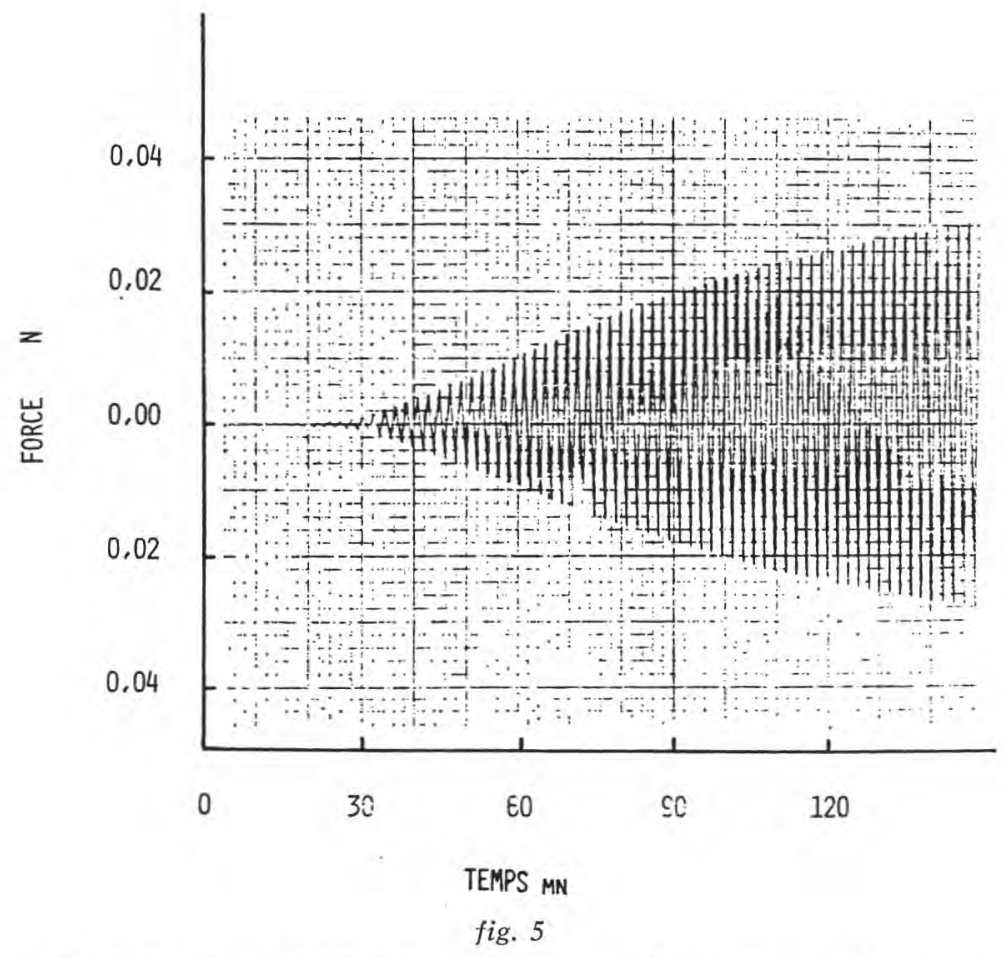

Evolution de la rigidité d'un gel obtenu à l'aide de carraghenates Conditions de coagulation :

Substrat : lait écrémé reconstitué ; matière sèche : $10 \% ; \mathrm{CaCl}_{2}, 2 \mathrm{H}_{2} \mathrm{O}$ : $10 \mathrm{~g} / 100 \mathrm{~kg}$ lait ; carraghenate : $0,3 \%$; traitement thermique : $82^{\circ} \mathrm{C}-20 \mathrm{~min}$; température de refroidissement : $20^{\circ} \mathrm{C}$.

Evolution of rigidity of skim-milk gel induced by addition Clotting conditions: of carrageenan

Subtsrate : reconstituted skim-milk; dried matter: $10 \%$; $\mathrm{CaCl}_{2} 2 \mathrm{H}_{2} \mathrm{O}$ : $10 \mathrm{~g} / 100 \mathrm{~kg}$ of milk; Carragenan: $0.3 \%$; thermal treatment: $82^{\circ} \mathrm{C}-20 \mathrm{~min}$; Cooling temperature: $20^{\circ} \mathrm{C}$.

l'absence de toute force d'inertie venant interférer avec la force exercée par le gel.

Dans la deuxième phase, lorsque le processus de gélification progresse, l'amplitude du mouvement sinusoïdal croît à chaque oscillation et passe par un maximum à chaque demi-période. Les points correspondant à ces maxima se situent, de part et d'autre de l'axe des temps, sur deux courbes d'allure sigmoïde, représentative de l'évolution caractéristique de la rigidité de chacun des types de gels étudiés. La tension du gel peut être ainsi quantifiée à tout 
moment par la valeur de la force exercée sur le mobile. Ainsi $60 \mathrm{mn}$ après apport de l'agent coagulant, la force exercée s'élève comme suit :

$\begin{array}{ccccc}\text { Agent coagulant } \ldots & \text { P } & \text { BS } & \text { BL } & \text { C } \\ \text { Force }(\mathrm{N}) \ldots \ldots \ldots & 0,084 & 0,058 & 0,036 & 0,0096\end{array}$

\section{Précision}

Compte tenu de l'étendue maxima de l'échelle $(0,2 \mathrm{~N} / 250 \mathrm{~mm})$ des graphes utilisés et de la précision de leur lecture $(0,5 \mathrm{~mm})$, la précision de la mesure se situe à $4 \cdot 10^{-4} \mathrm{~N}$. En portant l'étendue de mesure à $0,1 \mathrm{~N}$, pleine échelle, il est possible d'améliorer la précision à $2.10^{-4} \mathrm{~N}$.

\section{Répétabilité}

Sur la base d'une série de 40 mesures effectuées sur gel de type présure, le coefficient de variation est de $1,88 \%$. La répétabilité de la méthode est donc très satisfaisante.

\section{CONCLUSION}

La nouvelle méthode apparaît en raison de sa grande sensibilité, sa précision et sa répétabilité, bien adaptée à l'étude fine de la gélification du lait. Elle permet par ailleurs de réaliser des conditions d'essais harmoniques, de ne pas détruire la structure du gel et de quantifier les paramètres rhéologiques en unités exactes standard. Pour ces différentes raisons, elle présente un intérêt pour l'étude du comportement rhéologique des gels fragiles de biopolymères; par ailleurs, son principe peut être appliqué à la mise au point d'un capteur industriel pouvant être intégré sur une ligne mécanisée de fabrication. Ces deux voies de recherches sont poursuivies au laboratoire.

\section{Bibliographie}

Burnett (J.), Scott Blair (G. W.) (1963). - A speed compensated torsiometer for measuring the setting of milk by rennet. Dairy Ind., 28, 4, 220.

Frentz (R.) (1965). - Application de la thrombélastographie de Hartert à l'étude de la coagulation du lait. Le Lait, 45, 489-508.

Gervais (A.), CERF (O.) (1982). - Comparaison of two modes for fitting rennet induced milk coagulation kinetic. J. of Texture Studies (sous presse).

KowalchyK (A.W.) Olson (N.F.) (1978). - Firmness of enzymatically-formed gels measured by resistance to oscillatory deformation. J. Dairy Science, 61, 13751379.

RAMET (J. P.), Weber (F.) (1980). - Contribution à l'étude de l'influence des facteurs de milieu sur la coagulation enzymatique du lait reconstitué. Le Lait, $60,1-12$. 
Ramet (J. P.), El Mayda (E.), Weber (F.) (1982). - Influence of salting of reconstituted milk on curdling by rennet. J. of Textures Studies (accepté pour publication).

Schalinatus (E.), BehnKe (U.) (1967). - Untersuchung zur Wirkung von Labpreparaten verschiedener Herkunft. Milchwissenschaft, 22, 601-604.

Tarodo de La Fuente (B.), Frentz (R.) (1966). - Etude thrombélastographique de la coagulation du lait par la présure: action de la température et de la concentration en calcium. Le Lait, 46, 371-393.

Thomasow (J.), Voss (E.) (1977). - Methods for the determination of the firmness of milk coagulum. I.D.F. Annual Bull., Doc. $\mathrm{n}^{\circ} 99$.

VANDERHEIDEN (G.) (1976). - An apparatus for continously monitoring the structural rigidity of a gel. CSIRO Food. Res., 36, 45-48.

ZanNoni (M.), ANNibaldi (S.) (1981). - Standardization to the renneting ability of milk by Formagraph. Scienza e Technica Lattiero Casearia, 32, 79-94. 